\title{
The Short Baseline Near Detector
}

\author{
Joel A Mousseau*† \\ University of Michigan \\ E-mail: joelameumich.edu
}

SBND (Short-Baseline Near Detector) is a 112-ton liquid argon TPC neutrino detector under construction in the Fermilab Booster Neutrino Beam. Together with MicroBooNE and ICARUST600 detectors, SBND will search for short baseline neutrino oscillations in the $1 \mathrm{eV}^{2}$ mass range. SBND will also perform detailed studies of the physics of neutrino-argon interactions, thanks to a data sample of millions of electron and muon neutrino interactions. Finally, SBND plays a vital role in the on-going R\& D effort to develop the LArTPC technology, testing several technologies that can be used in a future kiloton-scale neutrino detectors for a long-baseline experiment. We will discuss the physics program, with a particular focus on the neutrino cross-section measurements and search for BSM physics as well as discuss the detector design and its current status.

The 39th International Conference on High Energy Physics (ICHEP2018)

4-11 July, 2018

Seoul, Korea

* Speaker.

$\dagger$ 
The goal of the Fermilab Short Baseline Neutrino program (SBN) is to resolve the present anomalies in accelerator and reactor experiments, and conclusively prove or dis-prove the sterile neutrino hypothesis. To accomplish this, the SBN program employs 3 neutrino detectors, each using liquid argon time projection chambers (LArTPCs). Each detector sits on-axis in the Fermilab booster neutrino beam (BNB), which provides a broad-band beam of neutrinos in the energy range of 0.1 to $3.0 \mathrm{GeV}$. Two of the detectors, MicroBooNE and ICARUS, have already been constructed. The third, the Short Baseline Near Detector (SBND) is currently being constructed and installed.

SBND is designed to serve as the near detector of the SBN program, and measure the unoscillated flux of $v_{\mu}$ s and intrinsic flux of $v_{e}$ s which make up the BNB. Such a measurement provides an indirect hypothesis for the expected $v_{\mu}$ flux at the ICARUS far detector. However, SBND provides much more vital information for the oscillation program, including measurements of neutrino-argon cross-sections, as well as measurements of nuclear effects in neutrino-argon interactions. Being the near detector, SBND is located $110 \mathrm{~m}$ from the BNB neutrino source, and observes the largest flux of neutrinos of the three detectors. As the near detector uses the same target media as the far detector, 112 tons of active liquid argon, direct conclusions may be made from the SBND data.

SBND consists of two drift regions of $2 \mathrm{~m}$, with a single mesh cathode in the center. The entire TPC is 5 meters long in the longitudinal direction. A diagram of the TPC is shown in Figure 1. The novel TPC design uses cold ADCs, pre-amplifiers, and motherboards connected to the end of each wire plane. Each anode plane is composed of three wire planes, with a $3 \mathrm{~mm}$ wire pitch. The TPC consists of twelve anode planes per drift region. The wires in each plane are connected across planes to reduce inactive regions. Wire winding of anode planes has begun in both the United States and the United Kingdom, and the first finished plane has arrived at Fermilab recently.

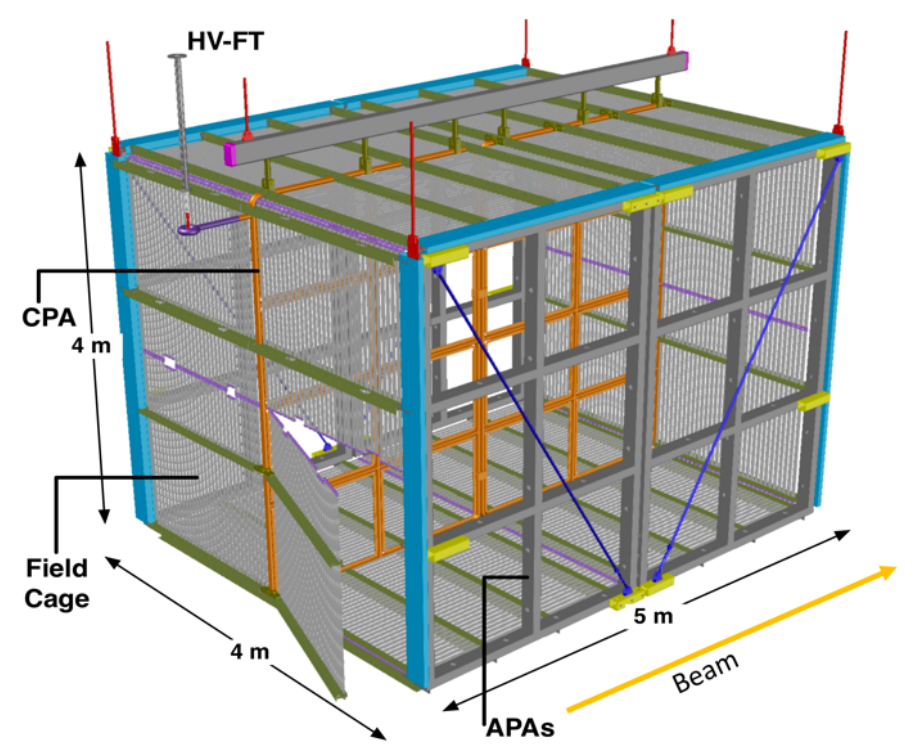

Figure 1: Three-dimensional model of the short baseline near detector TPC. The two drift regions are realized with two anode plane assemblies (APA), and one cathode plane assembly (CPA) in the center of the TPC. 
The entire TPC is encased in a stainless steel membrane cryostat, which is a similar design as the cryostat for the proto-DUNE experiment [1]. The TPC is supported from the cryostat roof, which contains the feedthroughs for signal and high voltage. Surrounding the cryostat are cosmicray tagging scintillator panels. These panels are responsible for reducing the largest background in LArTPCs, thoroughgoing and stopping cosmic rays. Due to the size of this background, the panels are designed to provide $4 \pi$ coverage around the cryostat. A set of panels has already been installed in the SBND building, and is currently taking data measuring interactions of the BNB with the surrounding building and earth.

Behind the anode planes, SBND plans to utilize and test multiple types of scintillation light detection. Argon scintillates in the near-ultraviolet spectrum, and as a consequence the wavelength must be shifter longer in order for it to be detectable by visible-light sensitive components. Scintillation light is a crucial element of reconstructing neutrino events in LArTPCs. This is because the drift time of electrons is very long (few ms) compared to the time of flight of particles produced in neutrino-argon interactions. By contrast, the scintillation light of these particles in liquid argon is very fast (few ns). This allows scintillation light to be used as a $t_{0}$, or start time of the electrons drifting toward the anode.

Visible light detection in SBND will primarily be accomplished using traditional, photomultiplier tubes (PMTs), as used in the MicroBooNE detector [2]. These PMTs are connected behind coated plates of tetraphenyl butadiene (TPB). However, two additional components of light detection have been approved for installation within SBND. The first are wavelength shifting plates located on the face of light-tight boxes called "Arapucas". A diagram of the Arapuca concept may be found in Figure 2. Additional light detection in SBND will be accomplished using TPB coated acrylic light bars. These light bars will be installed vertically in the same frames as PMTs and Arapucas. UV photons are converted to visible photons on the surface of the bars, which are totally internally reflected along the bar to the ends. Arrays of SiPMs are attached to the ends of light bars to detect the light. Digitization of the SiPM signals is accomplished with low-cost analog to digital converters (ADCs) designed to be used with medical ultrasound transducers. 96 light bars will be installed within SBND.

Finally, behind the cathode mesh of the TPC SBND plans to install reflective, TPB coated foils. Because the creation of scintillation photons in isotropic, locating all of the light-sensitive components on the anode side of the TPC misses photons which travel toward the cathode. Making the cathode reflective enables the recovery of some of these photons. The foils are coated with TPB via evaporation, so the UV light is shifted down to the visible before reflection. The ability to detect reflected light greatly increases the detected light yield of the PMTs, Arapucas and light bars, especially for tracks which originate near the cathode.

Once data taking begins, SBND will become a prolific source of neutrino-argon interactions. Simulations predict nearly 5 million charged-current $v_{\mu}$ events in three years of running. These further break down into 2 million charged-current events containing a single proton in the final state, and 1 millions events containing a single $\pi^{+} .2$ Millions neutral-current $v_{\mu}$ events are likewise expected in three years of running. Finally, $37 \mathrm{k}$ events are expected to be charged-current $v_{e}$ scattering from the intrinsic $v_{e}$ component of the BNB.

This wealth of neutrino-argon interaction data will prove to be very valuable in studying nuclear effects in neutrino-nucleus interactions, due to the large size of the argon nucleus. In partic- 




Figure 2: Demonstration of an Arapcua. UV photons are able to penetrate the second filter, denotated by the green line, but are unable to exit, resulting in visible photons being "trapped" in a reflective box. The trapped photons are eventually detected by arrays of SiPMs, shown on the top.

ular, SBN is interested in learning the intricacies of short range correlations, where the incoming neutrino interacts with a nucleon pair within the nucleus. These types of interactions have been observed in other LArTPC experiments, including ArgoNeut [3] and MicroBooNE, however their kinematics are currently not very well known. Additionally, final state interactions within the nucleus can change the energy, angle or charge of pions which exit in $\pi^{+}$events. SBND will have the ability to study these effects by analyzing the kinematics of outgoing $\pi^{+}$and $\pi^{0}$ events.

The short baseline near detector will provide a data-drive constraint on cross-section models necessary for detecting potential sterile neutrinos. It will likewise measure the intrinsic $v_{e}$ component of the BNB before any oscillations. Beyond the sterile and oscillation analysis, SBND is acting as a test-bed for new light detection technology. The novel cryostat design will springboard future TPC cryostat development. Finally, the intense neutrino beam provided by BNB will allow multiple cross-section analyses, and answer questions regarding short range correlations and final state effects. Exciting things are sure to be found once data taking begins in 2020 .

\section{References}

[1] B. Abi et al. [DUNE Collaboration], arXiv:1706.07081 [physics.ins-det].

[2] R. Acciarri et al. [MicroBooNE Collaboration], JINST 12, no. 02, P02017 (2017) doi:10.1088/1748-0221/12/02/P02017 [arXiv:1612.05824 [physics.ins-det]].

[3] R. Acciarri et al. [ArgoNeuT Collaboration], Phys. Rev. D 90, no. 1, 012008 (2014) doi:10.1103/PhysRevD.90.012008 [arXiv:1405.4261 [nucl-ex]]. 